\section{Manejo de la osteopenia en pacientes con artritis idiopatica juvenil}

\section{Resumen}

La enfermedad metabólica ósea pediátrica ha sido un tema secundario dentro del manejo de la Artritis Idiopática Juvenil. Si bien la enfermedad compromete a corto plazo la funcionalidad articular del niño, el desarrollo de osteopenia es un problema cuya frecuencia e impacto en el paciente no han sido objeto de suficiente atención. Es por ello que tanto el diagnóstico como el tratamiento de la osteopenia infantil en pacientes con Artritis Idiopática Juvenil suponen un problema para el clínico. La presente revisión pretende compilar la información disponible sobre estos dos temas y proponer un algoritmo de manejo y una agenda de investigación futura.

\section{Abstract}

Bone metabolic disease in children has been a secondary issue in the management of the Juvenile Idiopathic Arthritis. Although the disease produces joint functionality impairment in short term, the development of osteopenia is a problem which frequency and impact over the patient has not been subject of enough attention. Because of that, the diagnostic as well as treatment of infantile osteopenia in patients with Juvenile Idiopathic Arthritis mean a true challenge for the clinician. Present review aims to compile the available scientific information about both topics and to propose a management algorithm and a future research agenda.
Carlos Guillen Astete, María Luz Gamir Gami

Servicio de Reumatología

Unidad de Reumatología Pediátrica Hospital Universitario Ramón y Cajal

Correspondencia:

\section{Dr. Carlos Guillen Astete}

झ" cguillen.hrc@salud.madrid.org 


\section{Introducción}

La osteoporosis en la artritis idiopática juvenil (AIJ) es una definición que parte de la extrapolación de un concepto epidemiológico definido para población adulta. Su aplicación en población infantil es inapropiada por múltiples razones. La más importante radica en que la definición de osteoporosis corresponde al concepto histológico de osteopenia a la que se añade un perfil epidemiológico que teóricamente incrementa el riesgo de fractura en un individuo. Este perfil de riesgo no se puede definir con certeza por tres motivos: (1) En niños no se ha determinado con suficiente exactitud que estado densitométrico genera ese riesgo, (2) el esqueleto de un niño se encuentra en constante crecimiento y por lo tanto las técnicas habituales de medición subestiman el contenido mineral óseo y (3) Los instrumentos de medida de que disponemos no tienen patrones de comparación universalmente aplicables a población infantil y la generación de tablas regionales sigue siendo una tarea pendiente.

El retraso de la mineralización ósea y la reducción de su masa se considera un fenómeno multifactorial ampliamente descrito en pacientes con AIJ [1-3]. Los factores que influyen negativamente en la densidad mineral ósea son: La inflamación crónica [4-6],el retraso en la maduración puberal [3],malnutrición [7], debilidad muscular [6], actividad física limitada [8-9], inicio temprano de la AIJ [3], y corticoterapia [1-10].

En adelante utilizaremos el término osteopenia o enfermedad ósea asociada a la AlJ para referirnos al concepto histológico de disminución del grosor y la población de trabéculas asociado al adelgazamiento o no de la cortical en pacientes con esta enfermedad $[1,11,12]$.

La relación entre la osteopenia y AIJ desde un punto de vista fisiopatogénico ha evolucionado a partir del momento en que se reconoció dicha asociación epidemiológicamente. En la actualidad uno de los conceptos más relevantes en esta asociación es la interacción músculo-hueso y las alteraciones que sufre esta interacción durante los procesos inflamatorios crónicos [1,8,13,14].

Estas alteraciones no se presentan exclusivamente en los periodos en que la enfermedad está activa sino que se pueden documentar incluso en los periodos de remisión. Por esta razón la valoración del aparato musculoesquelético debe ser parte del estudio evolutivo del paciente con AlJ a lo largo de todo su seguimiento $[1,13,15]$.

Contra esta necesidad se presentan tres dificultades relevantes: Un escaso consenso en la recomendación de la técnica apropiada para evaluar la masa ósea, falta de definición terminológica para la osteoporosis en pediatría y una escasez de estudios controlados sobre intervenciones terapéuticas en pacientes con AIJ y osteoporosis.

\section{Definiciones previas}

\section{La densidad mineral ósea, masa mineral ósea y densidad ósea}

Los procesos biológicos relacionados con el crecimiento y remodelado óseo influyen en la geometría (grosor cortical, estructura trabecular) y densidad del hueso (unidad de masa por unidad de volumen). La resistencia del hueso está determinada por ambas variables $[12,13,16]$.

La formación de hueso se inicia con un depósito de matriz ósea orgánica sobre el que posteriormente se produce un proceso de mineralización [16].

La osteopenia como entidad patológica es el resultado de un insuficiente depósito de matriz orgánica o un incremento de la resorción ósea. Su correlato 
histológico es el de la reducción en el número de trabéculas óseas o en su grosor asociado o no a una disminución en el grosor de la cortical del hueso [16].

La osteomalacia es un desorden de la mineralización de la matriz ósea orgánica. Como resultado de este desorden la densidad mineral ósea se ve disminuida tanto a nivel trabecular como cortical. Este concepto permite ilustrar apropiadamente las deficiencias que tienen nuestros habituales sistemas de medida.

La densidad ósea se define como la masa mineral por volumen y se expresa en $\mathrm{g} / \mathrm{cm}^{3}$. Uno de los instrumentos más utilizados para valorar la densidad ósea es la radioabsorciometría de doble energía (DEXA). Los resultados obtenidos por medio de esta técnica son conocidos en la práctica clínica habitual como "densidad mineral ósea" pese a que expresa la masa ósea (mineral y orgánica) en gramos en relación con un área de hueso en $\mathrm{cm}^{2}$. Debido a ello es que una masa ósea reducida según una DEXA puede deberse indistintamente a osteopenia u osteomalacia [14].

Mientras que la densidad mineral ósea (DMO) debería expresar la masa mineral del hueso por unidad de volumen, nuestros instrumentos de medida habitual (DEXA) nos informan de la masa ósea detectada en un volumen de hueso demarcado por un área única a lo largo del espesor de una sección del mismo. Dicho volumen no es conocido por el aparato y por lo tanto, solamente, se expresa en unidad de área. La masa mineral ósea es la cantidad de materia inorgánica contenida en un hueso. En tanto que la masa mineral se concentra en la cortical del hueso, la masa mineral ósea es un buen marcador de resistencia a la fractura cuando la matriz es normal. La densidad ósea, por otro lado, es la medida de masa por unidad de volumen. Su valor es escaso en tanto que no discrimina entre ma- teria orgánica ni inorgánica. Determinados puntos de exploración pueden subsanar este problema: Si exploramos la cabeza del fémur la cortical será delgada mientras que si exploramos la diáfisis la cortical será gruesa $[11,13,14,18]$.

\section{La unidad osteo-muscular}

La tracción que realiza el sistema muscular sobre los huesos por medio de los tendones y sus entesis representan el estímulo más importante para el crecimiento del hueso y su mantenimiento [18]. Esto ha sido demostrado por medio de una correlación linear entre la masa muscular y la masa ósea en pacientes prepúberes, púberes y postpúberes [19].

Uno de los estudios que sirvieron para sustentar sólidamente la relación entre masa muscular y alteración de la geometría ósea fue el elaborado por Roth et al [8]. Los investigadores realizaron un estudio transversal sobre 57 pacientes con AlJ midiendo la masa muscular, densidad trabecular y el grosor cortical del hueso por medio de un método de tomografía cuantitativa periférica y comparando los resultados con las medias poblacionales para la misma edad y estatura. El estudio demostró que los pacientes con AlJ presentaban una significativa disminución en el área del corte transversal de la masa muscular del antebrazo, misma que se correlacionó linealmente con menor fuerza y menor grosor de la cortical del hueso. Aunque el tamaño de la población estudiada fue modesto se observó que la densidad trabecular se vio afectada en la AIJ poliarticular y la densidad cortical fue normal en todos los grupos de AlJ.

Los resultados de estas investigaciones denotan que los pacientes con AlJ sufren de una disminución en la masa ósea y por lo tanto, ésta, junto con la masa muscular y la fuerza motriz deben ser valoradas a lo largo de la evolución de estos pacientes. 
¿Por qué debemos ser cautos con la definición de osteoporosis infantil?

En adultos, la osteoporosis es una definición basada en el descenso de la masa ósea, alteración de la microarquitectura del hueso y aumento del riesgo de fracturas [20].Para establecerla se requiere comparar la masa ósea (medida por DEXA) del paciente con la de una persona joven. Por definición se exige que la medida del paciente se encuentre por debajo de -2.5 desviaciones estándar del patrón de referencia. Las mediciones cuya comparación se encuentren entre -1 y -2.5 desviaciones se consideran osteopenia. A esto se denomina la "escala T".

En el caso de niños, no existe una definición basada en la evidencia debido parcialmente al hecho de que no hay datos que estimen el riesgo de fractura basados en las desviaciones de la masa ósea [21]. Por otro lado, aunque dispusiéramos de tales datos, cualquier valoración de osteoporosis basada exclusivamente en la comparación de desviaciones respecto de un patrón (habitualmente la escala Z) será inferior a la que incluya además la medición de la masa y fuerza muscular. Aunque no hay un consenso internacional al respecto, Roth [1] estableció una serie de recomendaciones para el estudio de pacientes con AlJ en los que se haya demostrado una disminución de la masa ósea: Masa ósea en relación con la estatura, densidad trabecular y cortical, masa muscular en relación con estatura y masa ósea en relación con masa muscular.

\section{Intrumentos de valoración de la osteopenia en AIJ}

La DEXA es la técnica más comúnmente utilizada para valorar la densidad mineral ósea. Sus resultados se expresan en términos de masa mineral ósea (BMC, por sus siglas en inglés) en $\mathrm{g} / \mathrm{cm}^{2}$. Este es- tudio no mide realmente la densidad mineral ósea (que tendría que expresarse en $\mathrm{g} / \mathrm{cm}^{3}$ ), no expresa información sobre la matriz orgánica del hueso, y no diferencia entre hueso cortical y trabecular. Además los resultados se influencian por el tamaño del hueso y por lo tanto, por el tamaño del paciente.

La tomografía computarizada cuantitativa periférica ( $\mathrm{pQCT}$, por sus siglas en inglés) es un procedimiento que expone al paciente a menos de un tercio de la radiación requerida para realizar una DEXA. Este estudio permite distinguir el hueso trabecular del cortical, la densidad del hueso, la geometría ósea y la masa muscular. Esto la convierte en la prueba de elección para el estudio del hueso y músculo en pacientes pediátrico. Existen dos aspectos que restan aclarar sobre la utilidad del pQCT en el estudio del hueso en pacientes pediátricos: Su falta de precisión en la valoración de la cortical de niños pequeños y la extrapolación de los resultados del estudio óseo periférico al esqueleto axial. Esta última limitación podría ser omitida en tanto que varios estudios publicados a finales de la década de los 90s señalaban que la afectación ósea y la inducida por corticoides en pacientes con AlJ era fundamentalmente periférica $[2,22,23]$.

Finalmente el estudio del hueso por medio de la ultrasonografía no ha demostrado utilidad en la valoración de pacientes pediátricos con AlJ [24], por lo que su uso no está recomendado y hoy en día es muy infrecuente. Una excepción a ello es la sonometría ósea cuantitativa que en una serie pequeña de niños con AlJ demostró una aceptable correlación con la DEXA y cierto valor como herramienta de screening [25].

Schoenau et al [18] propusieron un algoritmo diagnóstico para distinguir los defectos metabólicos del hueso como primarios (dependientes de alteraciones en el depósito mineral o en la remodelación ósea) o secundarios (dependientes de alteraciones 
en la unidad músculo-ósea). Para su elaboración se requiere de una determinación de la fuerza muscular (o masa muscular) y del contenido mineral óseo (BMC, por sus siglas en inglés). La fuerza o masa muscular deben ser valorados en función de la estatura del paciente y el BMC se obtiene por medio de PQCT y se expresa en términos de gramos por $\mathrm{mm}$ de grosor de un voxel de $0.4 \times 0.4 \mathrm{~mm}$. Un defecto óseo primario debe sospecharse cuando en presencia de una masa muscular adecuada para la estatura el BMC no es adecuado. Por otro lado, un defecto secundario debe sospecharse cuando en un paciente con masa muscular inapropiada para la estatura se observa un BMC adecuado. El mismo grupo de autores han demostrado en pacientes sanos una correlación lineal entre la masa muscular y el BMC en niños y niñas.

Tabla 1. Ecuaciones de regresión que relacionan la masa muscular (CSA) y el contenido mineral óseo (BMC). Schoneau et al (J Bone \& Min Res; 2002[18]:6)

$\mathrm{BMC}_{\text {niñas }}(\mathrm{mg} / \mathrm{mm})=-6.60+3.03 \times \mathrm{CSA}\left(\mathrm{cm}^{2}\right) ; r=0.89$ $B M C_{\text {niños }}(\mathrm{mg} / \mathrm{mm})=+8.52+2.13 \times \mathrm{CSA}\left(\mathrm{cm}^{2}\right) ; r=0.92$

La masa muscular, medida en términos del área del corte transversal se correlaciona muy bien con la fuerza (mensurable mediante dinamometría). 15,18 Esto permite que la fuerza muscular pueda ser utilizada como medida para su aplicación en el razonamiento diagnóstico del algoritmo propuesto por Schoneau.

\section{Recomendaciones prácticas}

a. Se deben generar datos de referencia obtenidos por medio de los mismos instrumentos de medida (DEXA o pQCT) a utilizar en cada población.
Estos resultados deben estar disponibles según edad, sexo y etnia.

b. Los resultados deben valorarse en base a los correspondientes valores de referencia obtenidos y expresados en términos de veces de desviación estándar (Escala Z).

c. Se deben realizar correcciones por estatura en pacientes con retardo del crecimiento, y según estadio Tanner en caso de retraso del desarrollo puberal.

d. Una vez obtenidas las determinaciones de masa muscular y BMC se puede aplicar el algoritmo para determinar si la pérdida de masa ósea se debe a una menor masa muscular o es parte de un disturbio primario.

\section{Utilidad de los marcadores de metabolismo óseo}

\section{Pueden ser clasificados en tres grupos [26]:}

Marcadores generales: Calcio, fosfato, vitamina D, PTH, IGF-1.

Marcadores de formación ósea: Fosfatasa alcalina ósea, osteocalcina y procolágeno.

Marcadores de resorción ósea: Enzimas de osteoclásticas, productos de degradación del colágeno como la piridolina.

El mayor inconveniente del uso de estos marcadores es su disponibilidad para el seguimiento rutinario y la variabilidad circadiana de muchos de ellos. ${ }^{27}$ Por otra parte, muchos de los marcadores son específicos de la actividad osteoblástica u osteoclástica y por lo tanto no son útiles al momento de valorar la función remodeladora, modeladora o de formación de hueso, en la que ambos tipos de células actúan de forma simultánea. Escapan a estas debilidades las determinaciones de Vit D, de fosfatasa alcalina ósea y de paratormona. 


\section{RANK/RANKL}

El "receptor activador del factor nuclear kB" (RANK) y su correspondiente ligando RANKL son importantes coordinadores de la actividad de osteoblastos y osteoclastos. La proteína RANKL se expresa en la superficie de los osteoblastos y de células T activadas. Como resultado de su interacción con el receptor RANK, expresado por los osteoclastos, y sus precursores, se produce una maduración de estos y la prolongación de la supervivencia de las células maduras suprimiendo la apoptosis y por lo tanto, promoviendo el catabolismo óseo. El control de la actividad de este proceso depende en gran medida de la osteoprotegerina (OPG) que actúa secuestrando RANKL y evitando que ejerza su efecto sobre los osteoclastos. La OPG es sintetizada por los osteoblastos. En contra de la actividad de la OPG se encuentran: La deficiencia de estrógenos, la actividad de los glucocorticoides, el incremento de la activación de células T o la falta de estimulación mecánica. Todas estas situaciones o condiciones favorecen la actividad RANKL. Concretamente en pacientes con AlJ, la activación de células T (expresoras de RANKL), el incremento de IL-1, IL-6 y TNFalfa pueden inducir una activación sostenida de osteoclastos con subsecuente actividad catabólica del hueso. Por el contrario, la inhibición del TNF-alfa normalizaría el balance entre RANKL y OPG. ${ }^{28}$

\section{Epidemiologia de la enfermedad osteometabolica en AIJ}

Como se ha señalado al principio, existen múltiples condicionantes de la afectación patológica del hueso. La AlJ por si misma condiciona la disminución de la masa ósea por procesos como la activación de células T, liberación de IL-1 e IL-6, retardo del crecimiento y disminución de la actividad física. De forma indirecta, los pacientes con AlJ sufren además del efecto catabólico del hueso producido por los glucocorticoides y de la disminución de la masa muscular. En cierta medida, todos los factores condicionantes actúan de forma simultánea por lo que el abordaje de la afectación ósea en la AlJ debería ser integrándolos todos.

\section{Prevalencia}

En estudios transversales en los que predominantemente se utilizó la DEXA, se observó que los pacientes pertenecientes a todas las categorías de AlJ mostraron un descenso significativo de la masa ósea tanto en esqueleto axial como periférico [2]. En la mayor parte de estos estudios, sin embargo, no se realizó la corrección necesaria para pacientes con retraso del crecimiento, retraso puberal ni el correspondiente ajuste en función de la estatura. Pese a estas consideraciones, se ha observado que los pacientes con AlJ poliarticular presentan menor masa ósea que los pacientes con AlJ oligoarticular [2]. En estos grupos no se identificaron diferencias en el aporte de calcio y vitamina D ni tampoco se indentificó correlación alguna entre ambas variables y las relacionadas con el metabolismo óseo [2,22].

La masa ósea y la masa muscular fueron variables fuertemente correlacionadas [2].

Henderson et al, utilizando la DEXA como medida de masa mineral ósea determinó en una serie pequeña de prepúberes de ambos sexos una prevalencia de 30\% de sujetos con una $Z<-1.0 .{ }^{29} \mathrm{En}$ un estudio posterior únicamente con niñas postpúberes con el antiguo diagnóstico de artritis reumatoide juvenil y utilizando la misma herramienta de medición, encontró una prevalencia del 30\% [30]. En ambos estudios ningún paciente había estado expuesto a terapia con glucocorticoides. Aunque los tamaños muestrales fueron modestos, se alcan- 
zó identificar como factores de riesgo relacionados con una menor masa ósea a la mayor actividad de la enfermedad, mayor afectación articular y mayor limitación funcional [29]. Asimismo, los autores realizaron análisis de regresión logística para identificar la variable que contribuía con mayor peso a la disminución de la masa mineral ósea. En el análisis preliminar solamente la osteocalcina y la masa muscular tuvieron una correlación significativa $(p<0.05)$ sin embargo en el análisis "hacia delante", la osteocalcina no alcanzó significado estadístico. Por otro lado, la masa muscular explicó el cambio en la masa mineral ósea en un $76.3 \%$ con un coeficiente beta de -0.8093 .

Los estudios realizados con PQCT demuestran una reducción en la masa ósea en todos los subgrupos de pacientes con AlJ. Los cambios más Ilamativos son: reducción en el área del corte transversal del músculo, menor fuerza muscular, menor grosor de la cortical con preservación de su densidad y menor densidad trabecular yuxta articular (en articulaciones afectadas) $[8,11]$. Bechtold et al aplicó el uso de la pQCT en pacientes con AlJ y observó que, en general, a mayor duración de la enfermedad y a mayor gravedad menor estatura, menor densidad mineral ósea y peores parámetros fuerza ósea. En estos pacientes la estatura, el contenido mineral óseo y el área del corte transversal de la masa muscular fueron inferiores a los esperados por la edad sin embargo la masa muscular fue la apropiada para la estatura (defecto óseo primario) [11].

A lo largo de un seguimiento de 4 años de pacientes con AlJ, Roth et al identificó un incremento del área del corte transversal de la masa muscular y del grosor de la cortical. Este estudio fue el primero que demostró adaptaciones geométricas del hueso sin embargo estas modificaciones son el resultado de disturbios en el desarrollo esquelético a nivel medular y periosteal [13].

\section{Fracturas en pacientes con AIJ}

La prevalencia de fracturas en pacientes con AIJ es significativamente mayor que en población general. La serie de Elsasser et al [32] reportó 14 pacientes en de una cohorte de 70 niños, no obstante se trata de una publicación antigua y en la que no se contaba aún con las terapias biológicas. En otra cohorte posrterior, Murray et al [33] contabilizaron un $23 \%$ de fracturas patológicas en pacientes con AlJ. La mayor duración de la enfermedad marcada por un diagnóstico a menor edad, el retraso en el crecimiento (factor confusor conocido de disminución de la masa mineral ósea) y el uso de glucocorticoides fueron identificados como factores de riesgo.

La serie más grande publicada comparó la incidencia de fracturas en pacientes con AlJ. Se siguió 1939 sujetos con AlJ y fueron comparados con 207072 controles. La prevalencia de fracturas en el grupo con AlJ fue de $6.7 \%$ mientras que en el grupo control fue de 3.3\% [34]. Globalmente el sexo femenino se afectó con mayor proporción sin embargo una vez estratificados los pacientes en función de la edad, estas diferencias desaparecieron. La más reciente de las series de pacientes con AlJ seguidos para determinar la incidencia de fracturas vertebrales tras la iniciación de glucocoirticoides fue de 6 casos por cada 100 sujetos/año. La mayor parte de pacientes cursaron de forma asintomática. Los pacientes con fractura vertebral habían recibido una mayor dosis acumulada de glucocorticoides, habían tenido un mayor incremento en su índice de masa corporal y presentaban una mayor disminución de su densidad mineral ósea (escala Z) en los primeros 6 meses de tratamiento [35].

Parece razonable prestar especial atención a pacientes con AlJ poliarticular, sistémica y oligoarticular en los que no se haya conseguido alcanzar una rápida remisión y aquellos en los que se hayan utilizado glucocorticoides. En este grupo de pacientes 
conviene medir la densidad mineral ósea, realizar el ajuste en función de la estatura. Con esos resultados el clínico deberá considerar medidas de entrenamiento muscular o farmacológico.

\section{Los corticoides y la osteopenia en AIJ}

Múltiples estudios transversales han mostrado que el tratamiento con glucocorticoides se asocia a una disminución en la masa ósea del fémur y del esqueleto axial. Kotaniemi et al [23] realizó estimaciones de la masa ósea por medio de DEXA y el cálculo de la densidad volumétrica ósea y encontró que la asociación entre glucocorticoides y disminución de la masa ósea estaba mediada por un retraso en el crecimiento y una menor densidad volumétrica ósea. La más importante debilidad de este estudio radica en que los pacientes fueron controlados con sujetos pareados de por edad y sexo sanos en lugar en pacientes con AlJ no expuestos a corticoides.

En un estudio más reciente, Falcini et al [10] comparó la densidad mineral ósea medida por DEXA en pacientes con AlJ expuestos a glucocorticoides con pacientes con AlJ que recibieron AINEs. Sus resultados demostraron una significativa disminución de la BMD en el esqueleto axial de los pacientes que recibieron glucocorticoides. Este estudio se presta a pasar por alto el hecho de que los pacientes con cuadros de AlJ más graves tomarán con mayor probabilidad esteroides que aquellos en los que la enfermedad sea menos agresiva. No obstante ello, el trabajo aportó un hallazgo importante: El uso de suplemento de Vitamina D y Calcio no modificó el efecto de los corticoides en la densidad mineral ósea en los pacientes con AlJ. El retardo del crecimiento inducido por los glucocorticoides exige que se hagan correcciones al momento de valorar la escala Z en función de la estatura.
No existen estudios dirigidos a determinar si los medicamientos modificadores de la enfermedad producen cambios en la masa mineral ósea con excpeción de un estudio prospectivo en el que se demostró que la administración de Metotrexate no se asociaba a ningún tipo de modificación. ${ }^{31}$ Por otro lado el efecto que ejercen los anti-TNF alfa inhibiendo rapidamente la inflamación en pacientes con AlJ y evitando la interacción de la proteina RANK y su lingando. ${ }^{28}$

\section{Alternativas terapéuticas}

\section{Calcio y vitamina D}

El desarrollo del hueso depende en primer lugar del depósito de la matriz orgánica y en segundo lugar de la mineralización. Los déficits de calcio producen raquitismo como consecuencia de la alteración de los cartílagos de crecimiento y osteomalacia en otros puntos del esqueleto.

Entendida histológicamente la osteopenia como la reducción en el número y grosor de las trabéculas y como el adelgazamiento de la cortical, esta no produce por una alteración de la mineralización sino por una alteración del depósito de la matríz [16].

No se ha demostrado correlación entre el aporte de calcio y las variables óseas en niños sanos con un aporte mínimo diario de 500 mg de calcio. Esto supone que una vez alcanzado este requerimiento mínimo, los aportes suplementarios no supondrían un beneficio adicional [16].

Teniendo en cuenta esto y que además lo que de forma corriente se entiende por "osteoporosis" en pacientes con AlJ realmente es osteopenia se concluye que el uso de suplementación con calcio y vitamina $D$ con fines profilácticos tiene sustento limitado una vez que el paciente ha alcanzado un aporte mínimo. 
Por otro lado, ha sido ampliamente reconocido que las enfermedades crónicas cursan con deficiencias de vitamina D [36]. Finalmente, cabe anotar que la terapia con glucocorticoides pueden interferir con el metabolismo de vitamina $\mathrm{D}$ y calcio como consecuencia de una reducción de la absorción de calcio e induciendo a un hiperparatiroidismo secundario.

La suplementación de Vitamina D ha sido estudiada en escasos trabajos y los resultados, una vez contrastados resultan controversiales. En un estudio prospectivo con pacientes con AlJ suplementados con vitamina $D$ no se identificó beneficio en términos de densidad mineral ósea medida por DEXA [37]. No obstante, en niños con AlJ y deficiencia de vitamina D, la suplementación con calcitriol produjo incrementos significativos en la densidad mineral ósea [38]. Un estudio reciente, controlado y randomizado administró 1000 mg de Calcio y 400 U de Vitamina D a pacientes con AlJ demostrando puntuales beneficios estadísticamente significativos en términos de BMD [39].

La guía de la sociedad europea de endocrinología pediátrica señala que existe "escasa" evidencia que sustente el uso profiláctico de Vitamina D en la prevención o el tratamiento de la osteoporosis inducida por glucocorticoides. En términos de recomendación solamente se incide en un adecuado aporte de calcio y vitamina D, y la vigilancia de potenciales estados carenciales [36].

\section{Bifosfonatos}

El principal efecto de los bifosfonatos consiste en la supresión del metabolismo de los osteoclastos. Los efectos colaterales incluyen la hipocalcemia postinfusional, hipofosfatemia, cefalea, efectos gastrointestinales y síntomas catarrales. La supresión del remodelado óseo es temporal y dependiente de la vigencia de la posología. La reparación de fracturas puede alterarse, no así el crecimiento.
No existen muchos estudios protocolizados para medir el beneficio del uso de bifosfonatos en pacientes con AlJ. Thornton et al [40] realizaron una revisión sistemática de la escasa literatura disponible. Un ensayo clínico y tres estudios prospectivos demostraron incremento de la densidad mineral ósea respecto de la situación basal con cambios significativos pero no realizaron comparativas directas con los controles. La heterogeneidad de las variables e instrumentos de medición imposibilitó la obtención de una n combinada.

A diferencia de los pacientes con osteogénesis imperfecta, los pacientes con AlJ presentan un déficit óseo fundamentalmente secundario por lo que el tratamiento con bifosfonatos no tendría porque ser de primera elección. Su uso podría estar restringido a pacientes con fracturas en el contexto de un decenso generalizado, refractario y significativo de la masa ósea refractario al tratamiento de la enfermedad. No tiene lugar indicación alguna basada en el uso de un patrón Z en pediatría de la misma manera que en adultos se usa el patrón $T$ y cuyo punto de corte viene definido por la casuística de fracturas patológicas [1].

\section{Entrenamiento muscular}

La relación directa entre formación de hueso y fuerza muscular ha sido demostrada fehacientemente en población pediátrica y adolescentes [12]. Esta se debe a que la mayor carga mecánica a la que está expuesto el hueso es la generada por la tensión muscular por encima de otras fuerzas como la compresión por el peso corporal o las fuerzas rotacionales. Los pacientes con AIJ presentan una disminución en sus capacidades aeróbicas y anaeróbicas probablemente relacionado con un menor entrenamiento muscular y que se manifiesta en un incremento considerable del oxígeno [41]. 


\begin{tabular}{|c|c|c|c|}
\hline Periodo & Vigilancia & Situación de alerta & Medida \\
\hline Diagnóstico & $\begin{array}{l}\text { Talla, peso, velocidad de } \\
\text { crecimiento, IMC } \\
\text { Niveles de Vit D, PTH } \\
\text { condicional. }\end{array}$ & $\begin{array}{l}\text { Ralentización de la velocidad de } \\
\text { crecimiento, menor talla para el peso/ } \\
\text { edad. }\end{array}$ & $\begin{array}{l}\text { Suplir deficiencias nutricionales. } \\
\text { Tratamiento intenso de la AlJ. }\end{array}$ \\
\hline Seguimiento & $\begin{array}{l}\text { velocidad de crecimiento, } \\
\text { fuerza muscular (pQCT) }\end{array}$ & $\begin{array}{l}\text { Menor fuerza muscular para la talla } \\
\text { o ralentización de la velocidad de } \\
\text { crecimiento. }\end{array}$ & $\begin{array}{l}\text { Intensificar el tratamiento AlJ. } \\
\text { Potenciar ejercicio anaeróbico } \\
\text { > aeróbico. }\end{array}$ \\
\hline En remisión & $\begin{array}{l}\text { velocidad de crecimiento, } \\
\text { fuerza muscular (pQCT) }\end{array}$ & $\begin{array}{l}\text { Menor fuerza muscular para la talla } \\
\text { o ralentización de la velocidad de } \\
\text { crecimiento. }\end{array}$ & $\begin{array}{l}\text { Potenciar ejercicio anaeróbico } \\
\text { > aeróbico. }\end{array}$ \\
\hline
\end{tabular}

La unidad osteo-muscular debe ser el eje del tratamiento y profilaxis de la pérdida de masa ósea. Por un lado el entrenamiento con ejercicio anaeróbico aumenta la resistencia muscular y la capacidad aeróbica sin embargo no aumenta la fuerza muscular y sin ello no se produce beneficio en el hueso. El incremento de la fuerza muscular por medio de entrenamiento anaeróbico determina mayor exposición ósea a tensiones y ello estimula la formación de hueso. La capacidad para sostener estas fuerzas depende de la resistencia muscular y por lo tanto el entrenamiento debe hacerse en ambos tipos de ejercicio [22].

Es recomendable incrementar la indicación de ejercicio físico aeróbico y anaeróbico en los pacientes con AlJ por lo que el manejo agresivo de la inflamación debe instaurarse tan pronto sea posible evitando que se produzcan alteraciones articulares que impidan este entrenamiento. Por otro lado y salvo que existan datos de inestabilidad articular, no debería indicarse la restricción de deportes de alto impacto en pacientes con AlJ.

\section{Recomendaciones}

Teniendo en cuenta todo lo anteriormente señalado vamos a elaborar una propuesta de recomendaciones de manejo protocolizado de la enfermedad osteometabólica en pacientes con AIJ.

\section{¿Cuándo indicar una medida de la masa mineral ósea?}

Al igual que cualquier otra prueba complementaria, la solicitud de una DMO (idealmente una PQCT) debe tener por finalidad una toma de decisión concurrente.

La solicitud de una DEXA se justificaría en los siguientes casos:

I. AlJ Oligoarticular que no alcanza la remisión.

II. AIJ Poliarticular o sistémica con baja estatura para la edad o con ralentización de la velocidad de crecimiento.

III. Cualquier paciente que haya tenido una fractura

\section{¿Qué hacer con los resultados de la prueba?}

Lo primero es que los resultados estén ajustados a la estatura del paciente y según ello procederemos de la siguiente forma:

a. Si Z > -1.0 desviaciones estándar: Medidas anaeróbicas > aeróbicas e intensificación del tratamiento de la enfermedad. Control dinamométrico en 6 meses y DMO en 1 año.

b. Si $Z<-1.00>-1.0$ sin variación en un año pese a intensificación de tratamiento de AlJ + ejerci- 
cios: Cambio de tratamiento de la enfermedad de base (considerar fracaso terapéutico) y ejercicio físico anaeróbico $>$ aeróbico.

\section{c. Si $Z<-2.5$. Actuar como en el caso anterior y valorar el uso de bifosfonatos.}

\section{Referencias}

1. Roth J, Bechtold S, Borte G, Dressler F, Girschick HJ, Borte M. Osteoporosis in juvenile idiopathic arthritis--a practical approach to diagnosis and therapy. Eur J Pediatr. 2007;166(8):775-84.

2. Pepmueller PH, Cassidy JT, Allen SH, Hillman LS. Bone mineralization and bone mineral metabolism in children with juvenile rheumatoid arthritis. Arthritis Rheum. 1996;39(5):74657.

3. Stagi S, Masi L, Capannini S, Cimaz R, Tonini G, Matucci-Cerinic $M$, et al. Cross-sectional and longitudinal evaluation of bone mass in children and young adults with juvenile idiopathic arthritis: the role of bone mass determinants in a large cohort of patients. J Rheumatol. 2010;37(9):1935-43.

4. Gravallese EM. Bone destruction in arthritis. Ann Rheum Dis. 2002;61 Suppl 2:ii84-86.

5. Strand V, Kavanaugh AF. The role of interleukin-1 in bone resorption in rheumatoid arthritis. Rheumatol Oxf Engl. 2004;43 Suppl 3:iii10-iii16.

6. Burnham JM. Inflammatory diseases and bone health in children. Curr Opin Rheumatol. 2012;24(5):548-53.

7. Cleary AG, Lancaster GA, Annan F, Sills JA, Davidson JE. Nutritional impairment in juvenile idiopathic arthritis. Rheumatol Oxf Engl. 2004;43(12):1569-73.

8. Roth J, Palm C, Scheunemann I, Ranke MB, Schweizer R, Dannecker GE. Musculoskeletal abnormalities of the forearm in patients with juvenile idiopathic arthritis relate mainly to bone geometry. Arthritis Rheum. 2004;50(4):1277-85.

9. Häkkinen A, Sokka T, Kautiainen $H$, Kotaniemi A, Hannonen P. Sustained maintenance of exercise induced muscle strength gains and normal bone mineral density in patients with early rheumatoid arthritis: a 5 year follow up. Ann Rheum Dis. 2004;63(8):910-6.

10. Falcini F, Trapani S, Civinini R, Capone A, Ermini M, Bartolozzi $G$. The primary role of steroids on the osteoporosis in juvenile rheumatoid patients evaluated by dual energy X-ray absorptiometry. J Endocrinol Invest. 1996;19(3):165-9.

11. Bechtold S, Ripperger P, Dalla Pozza R, Schmidt H, Häfner R, Schwarz HP. Musculoskeletal and functional muscle-bone analysis in children with rheumatic disease using peripheral quantitative computed tomography. Osteoporos Int J Establ Result Coop Eur Found Osteoporos Natl Osteoporos Found USA. 2005;16(7):757-63.
12. Schoenau E, Frost HM. The «muscle-bone unit» in children and adolescents. Calcif Tissue Int. 2002;70(5):405-7.

13. Roth J, Linge M, Tzaribachev N, Schweizer R, KuemmerleDeschner J. Musculoskeletal abnormalities in juvenile idiopathic arthritis--a 4-year longitudinal study. Rheumatol Oxf Engl. 2007;46(7):1180-4.

14. Rauch F, Schoenau E. Changes in bone density during childhood and adolescence: an approach based on bone's biological organization. J Bone Miner Res Off J Am Soc Bone Miner Res. 2001;16(4):597-604.

15. Specker BL, Schoenau E. Quantitative bone analysis in children: current methods and recommendations. J Pediatr. 2005;146(6):726-31.

16. Schoenau E, Saggese G, Peter F, Baroncelli Gl, Shaw NJ, Crabtree NJ, et al. From bone biology to bone analysis. Horm Res. 2004;61(6):257-69.

17. Hoyer-Kuhn H, Semler O, Schoenau E, Roschger P, Klaushofer K, Rauch F. Hyperosteoidosis and hypermineralization in the same bone: bone tissue analyses in a boy with a homozygous BMP1 mutation. Calcif Tissue Int. 2013 Dec;93(6):565-70.

18. Schoenau E, Neu CM, Beck B, Manz F, Rauch F. Bone mineral content per muscle cross-sectional area as an index of the functional muscle-bone unit. J Bone Miner Res Off J Am Soc Bone Miner Res. 2002;17(6):1095-101.

19. Schiessl H, Frost HM, Jee WS. Estrogen and bone-muscle strength and mass relationships. Bone. 1998;22(1):1-6.

20. Assessment of fracture risk and its application to screening for postmenopausal osteoporosis. Report of a WHO Study Group. World Health Organ Tech Rep Ser. 1994;843:1-129.

21. Chan YY, Bishop NJ. Clinical management of childhood osteoporosis. Int J Clin Pract. 2002;56(4):280-6.

22. Kotaniemi A, Savolainen A, Kröger $H$, Kautiainen $H$, Isomäki $H$. Weight-bearing physical activity, calcium intake, systemic glucocorticoids, chronic inflammation, and body constitution as determinants of lumbar and femoral bone mineral in juvenile chronic arthritis. Scand J Rheumatol. 1999;28(1):19-26.

23. Kotaniemi A, Savolainen A, Kautiainen H, Kröger H. Estimation of central osteopenia in children with chronic polyarthritis treated with glucocorticoids. Pediatrics. 1993;91(6):1127-30.

24. Scheunemann I, Dannecker GE, Roth J. Phalangeal bone ultrasound is of limited value in patients with juvenile idiopathic arthritis. Rheumatol Oxf Engl. septiembre de 2006;45(9):1125-8.

25. Hartman C, Shamir R, Eshach-Adiv O, losilevsky G, Brik R. Assessment of osteoporosis by quantitative ultrasound versus dual energy X-ray absorptiometry in children with chronic rheumatic diseases. J Rheumatol. mayo de 2004;31(5):981-5.

26. Roth J, Bechtold S, Borte G, Dressler F, Girschick HJ, Borte M. Osteoporosis in juvenile idiopathic arthritis--a practical approach to diagnosis and therapy. Eur J Pediatr. agosto de 2007;166(8):775-84.

27.Rauchenzauner M, Schmid A, Heinz-Erian P, Kapelari K, Falkensammer G, Griesmacher A, et al. Sex- and age-specific 
reference curves for serum markers of bone turnover in healthy children from 2 months to 18 years. J Clin Endocrinol Metab. febrero de 2007;92(2):443-9.

28. Hofbauer LC, Schoppet M. Clinical implications of the osteoprotegerin/RANKL/RANK system for bone and vascular diseases. JAMA J Am Med Assoc. 28 de julio de 2004;292(4):490-5.

29. Henderson CJ, Cawkwell GD, Specker BL, Sierra RI, Wilmott RW, Campaigne BN, et al. Predictors of total body bone mineral density in non-corticosteroid-treated prepubertal children with juvenile rheumatoid arthritis. Arthritis Rheum. noviembre de 1997;40(11):1967-75

30. Henderson CJ, Specker BL, Sierra RI, Campaigne BN, Lovell DJ. Total-body bone mineral content in non-corticosteroidtreated postpubertal females with juvenile rheumatoid arthritis: frequency of osteopenia and contributing factors. Arthritis Rheum. marzo de 2000;43(3):531-40.

31. Bianchi ML, Cimaz R, Galbiati E, Corona F, Cherubini R, Bardare $M$. Bone mass change during methotrexate treatment in patients with juvenile rheumatoid arthritis. Osteoporos Int J Establ Result Coop Eur Found Osteoporos Natl Osteoporos Found USA. 1999;10(1):20-5.

32. Elsasser U, Wilkins B, Hesp R, Thurnham DI, Reeve J, Ansell BM. Bone rarefaction and crush fractures in juvenile chronic arthritis. Arch Dis Child. mayo de 1982;57(5):377-80.

33. Murray K, Boyle R, Woo P. Pathological fractures and osteoporosis in a cohort of 103 systemic onset juvenile idiopathic arthritis patients. Arthritis Rheum. 2000;43:S119.

34. Burnham JM, Shults J, Weinstein R, Lewis JD, Leonard MB. Childhood onset arthritis is associated with an increased risk of fracture: a population based study using the General Practice Research Database. Ann Rheum Dis. agosto de 2006;65(8):1074-9.

35. Rodd C, Lang B, Ramsay T, Alos N, Huber AM, Cabral DA, et al. Incident vertebral fractures among children with rheumatic disorders 12 months after glucocorticoid initiation: a national observational study. Arthritis Care Res. enero de 2012;64(1):12231.

36. Hochberg Z, Bereket A, Davenport M, Delemarre-Van de Waal HA, De Schepper J, Levine MA, et al. Consensus development for the supplementation of vitamin $D$ in childhood and adolescence. Horm Res. 2002;58(1):39-51.

37.Reed A, Haugen M, Pachman LM, Langman CB. 2 5-Hydroxyvitamin $D$ therapy in children with active juvenile rheumatoid arthritis: short-term effects on serum osteocalcin levels and bone mineral density. J Pediatr. octubre de 1991;119(4):657-60.

38. Cimaz R. Osteoporosis in childhood rheumatic diseases: prevention and therapy. Best Pract Res Clin Rheumatol. 2002;16(3):397-409.
39. Lovell DJ, Glass D, Ranz J, Kramer S, Huang B, Sierra RI, et al. A randomized controlled trial of calcium supplementation to increase bone mineral density in children with juvenile rheumatoid arthritis. Arthritis Rheum. 2006;54(7):2235-42.

40. Thornton J, Ashcroft D, O'Neill T, Elliott R, Adams J, Roberts C, et al. A systematic review of the effectiveness of strategies for reducing fracture risk in children with juvenile idiopathic arthritis with additional data on long-term risk of fracture and cost of disease management. Health Technol Assess Winch Engl. 2008;12(3):iii-ix, xi-xiv, 1-208.

41. Takken T, Hemel A, van der Net J, Helders PJM. Aerobic fitness in children with juvenile idiopathic arthritis: a systematic review. J Rheumatol. 2002;29(12):2643-7.

\section{Opina sobre este artículo:}

\section{f 8 in $8+\mathbf{S}$ t}

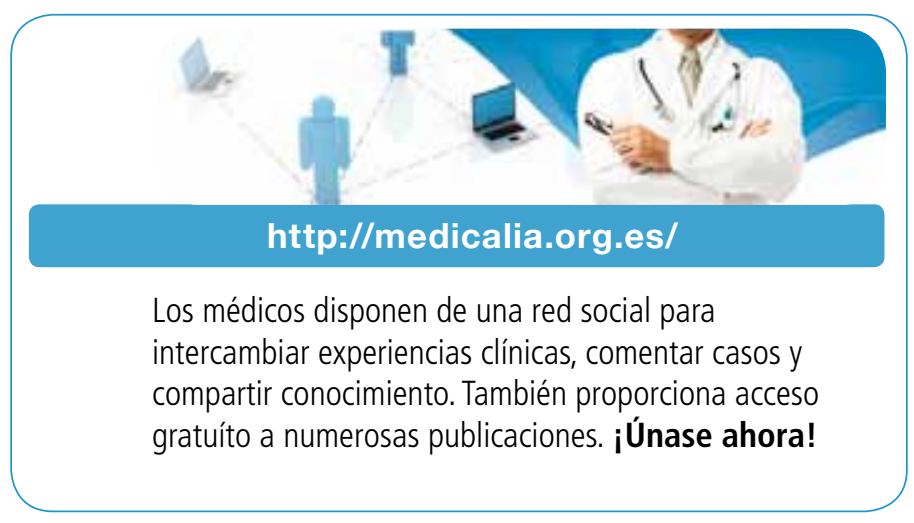

\section{Publish with iMedPub}

http://www.imed.pub

Acta Reumatológica es una revista que tiene por fin la difusión de estudios clínicos relacionados con aspectos prácticos del diagnóstico, tratamiento y seguimiento de pacientes con patología reumatológica, de estudios epidemiológicos relacionados con patología inflamatoria y musculoesquelética de presentación común o infrecuente en la práctica clínica tanto en población adulta como pediátrica, de casos clínicos de patología poco habitual o de presentaciones inhabituales de patología frecuente, de imágenes didácticas e ilustrativas en reumatología y del estado actual e innovación en la formación especializada en reumatología. 